\title{
A STUDY ON DEVELOPING A SMART ENVIRONMENT IN AGRICULTURAL IRRIGATION TECHNIQUE
}

\author{
Angel $\mathrm{C}$ and Asha S \\ School of Computing Science and Engineering, VIT University, Chennai, Tamil Nadu
}

\begin{abstract}
Maintaining a good irrigation system is a necessity in today's water scarcity environment. This paper describes a new approach for automated Smart Irrigation (SIR) system in agricultural management. Using various types of sensors in the crop field area, temperature and moisture value of the soil is monitored. Based on the sensed data, SIR will automatically decide about the necessary action for irrigation and also notifies the user. The system will also focus on the reduction of energy consumption by the sensors during communication.
\end{abstract}

\section{KEYWORDS}

Smart Irrigation, Intelligent Irrigation, Precision Agriculture

\section{INTRODUCTION}

The development in smart environmental technology and the application of Wireless Sensor Networks (WSNs) creates a new way of research in the field of agriculture. Now-a day sensor technologies are very much helpful in creating the smart précised environment. Using this technique, the WSN deployments for various farming applications in the Indian as well as global scenario are surveyed. Also the possibility of using this technique for the crop growth starting from its cultivation to harvesting is analyzed. In this way, various parameters needed for particular crop maintenance is focused and the problems for improvement and future development are considered.

Agriculture plays a vital role in the development of country's economy. The daily need for food shows the importance of agricultural development. Growing a particular crop in a particular region takes the privilege of monitoring the growth from cultivation till harvesting. One of the main challenges in agricultural activities is irrigation. As the global climate decreases the source of water throughout the world, it is necessary to take steps for preserving it. However traditional irrigation management is done by the people itself. It requires the presence and continuous monitoring of irrigation by the farmers in the field area. The user must need to manually change the direction of water flow using large pipes in the field. It creates a need for more labour work and maintenance. A little advancement in traditional system involves in establishing centralized control of irrigated land [3] using wired architecture which will also leads to more cost and maintenance manually. Hence a new system SIR which makes the irrigation system fully automated with the development of Wireless Sensor Actuator Networks (WSAN) is developed. 
International Journal of Ambient Systems and Applications (IJASA) Vol.3, No.2/3, September 2015

In India, the method of cultivation of paddy in a particular region depends largely on factors such as situation of land, type of soils, irrigation facilities and availability of moisture level and distribution of rainfalls. Paddy fields are the typical feature of rice farming in east, south and southeast Asia. Paddy crops can be built into steep hillsides and adjacent to depressed or steeply sloped features such as rivers or marshes. They can require a great deal of labour and materials to create, and need large quantities of water for irrigation. Oxen and water buffalo, adapted for life in wetlands, are important working animals used extensively in paddy field farming.

The agriculture systems in developing countries are still labour dependent and do not use any crop management, pest/disease control or quality management systems. The traditional irrigation systems in agriculture use uniform water distribution in fields at regular intervals, which is not optimal. Hence, a technology based agricultural monitoring system which decides itself intelligently and performing the action is needed.

In this paper, for developing a smart thinking irrigation system, various WSN applications and intelligent technique is reviewed. To focus on the specific requirements, the devices, sensors and communication techniques associated with WSNs in agricultural applications are analyzed comprehensively. We present various related work to thoroughly explore the existing solutions proposed in the literature in various categories according to their design and implementation related parameters.

\subsection{Paper organization}

The rest of the paper is organized as follows. Section 2 describes about various techniques and methods used for irrigating the field area and the issues faced by it. Design of a SIR system for sprinkler irrigation method in agricultural application is discussed in Section 3. Discussion about various parameters which needed to be processed in future is given in Section 4. And finally, the paper concludes in Section 5.

\section{RELATED WORK}

In the process of developing an automated wirelessly monitored irrigation system, several methods were followed.

\subsection{Remote Monitoring using GSM}

The subscriber or user sends activation command to the system via SMS. The system [1] will check the moisture level and if it is less than the prescribed level, the system will start the motor. While the motor is running, the system will monitor the soil moisture and water level constantly. If the moisture level reaches the sufficient level, the motor will be turned off. The corresponding event of the operation of motor is notified to the user using SMS. This motor is controlled by a starter which is indirectly activated by a relay circuit. Using GSM technique, an automated remote monitored irrigation system is provided. The system sets the time period depending on the temperature and humidity of the soil for irrigating the land. The humidity and temperature level of the soil and the crop varies for various types of crops.

\subsection{Monitoring using fuzzy controller}

The system [2] consists of two units, WSN and a monitoring center. Nodes in the monitoring area collect information of the soil moisture and growth information of different crops in different 
International Journal of Ambient Systems and Applications (IJASA) Vol.3, No.2/3, September 2015

periods using solar power. Wireless Sensor Network (WSN) contains many sensor nodes and controller nodes. The data sensed from the WSN is given as inputs to the monitoring center which in turn gives the information about the irrigation, demand of the water level and control over the opening or closing of the valves. Zigbee network in a mesh network topology is used to meet the network coverage and reduce the energy consumption and the cost. Some of the nodes are assigned as routers and coordinators. Sensor nodes sense the temperature and humidity whereas the routing nodes route the communication information and forwarding the data to the relevant nodes and the coordinator node receives data from the routing nodes and sent it to host computer monitoring center through RS232 serial bus. The monitoring center record the real time moisture value form all the nodes and calculate crop irrigation water requirement and output the result to WSN and control to the valves. The soil moisture sensor TDR-3A is used for measuring the moisture value.

\subsection{Monitoring using humidity sensors}

For measuring the humidity value of a region, SY-HS-220 humidity sensor [5] is used. It works on the operating temperature range of $0-60 \mathrm{o}$. Temperature value is sensed by the sensor LM35IC in which the output voltage is linearly proportional to the Celsius temperature. TinyOS [9] is an open source component based low power operating system which is designed only for embedded system application. TinyOS based IRIS motes is used to measure the moisture level of paddy field and MD100CB sensor motes are used to reduce the number of motes used. A low cost soil moisture sensor [6] is used to control water supply in water deficient areas. The sensor communicates the information via XBEE wireless communication module to a centralized server. The server provides the control to the water supply and displays the moisture data in a more user friendly interface. Two galvanized metallic electrodes are kept at a distance of $30 \mathrm{~mm}$ inside the soil area in an acrylic sheet. The change of impedance between two galvanized metallic electrodes due to the varying moisture content in the surrounding medium is measured. Based on all these measurement, the data is transferred to the server for processing.

\subsection{Monitoring in drip irrigation}

In order to achieve the effective usage of water in drip irrigation [10, 7], sensors are scattered throughout the field region. Sensors are used to sense the water level in the well as well as in the water tank. If the measured value is less than the prescribed value, then alert is given to the user. If the level of the water in the tank is low, then it directs the command to the nearby well. The pump is activated thereby the valve is opened and the water is pumped out to the well. Sensors in the field sensed the value of temperature and moisture in the field and based on the input value, the system decides either to open the valve or not.

\subsection{Remote Monitoring using sensors}

In the irrigation monitoring system [11,12], the information regarding soil moisture, temperature etc are sensed by the Bluetooth wireless transmitters and the time specific decision for irrigation is made according to the information sensed. The irrigation control unit gets the decision and sends the position of the irrigation system using GPS receiver to the base station through real time monitoring. Base station in turn sends control signals to the irrigation control station to operate the device for water usage. Also distributed irrigation system [13], sensor based irrigation system [14] and automated field-specific irrigation system [15] are also provided good irrigation control. Yet, all these do not consider the pollution of water. 


\section{PROPOSED METHOD}

The proposed method discusses about the idea of developing a Smart Irrigation (SIR) using wireless sensors. The system also focuses on reducing the cost and energy consumption during the process. The entire field is embedded with sensor nodes including soil moisture sensors, humidity sensors, soil $\mathrm{Ph}$ sensor, controller node, solar panels, irrigation sprinkler and control valves. The overall system will be activated for every 1 hour. As the timer triggers, the sensor will sense the data and communicate it to the nearby controller node. The controller node acts as a router and sends the data to the server. The controller node communicates the information once all the information is ready thereby reducing the power consumption. Figure 1 will describe the overview of SIR system functionalities.



Figure 1: Overview of SIR system

If the moisture value and the humidity value is less than the threshold prescribed then the system will decide to open the valves. The valves in the pipe will open for only 3 seconds and then automatically closed. As the entire system will be triggered for every 1 hour, it is more sufficient for a plant to maintain the moisture required for it.

Also the water level sensor in the tank will monitor the water level inside the tank and if it is lower than the necessary parameter, the system will start the motor to pump the water from the well. For all the events, the information about the status of the water level, motor on/off, moisture and temperature level is notified to the user via SMS. Since all the nodes are powered by solar energy from the solar panel, the SIR system will reduce the energy supply problem also.

The following flowchart will define the detailed steps involved in the process of SIR system. The system also focused on minimizing the cost for communication between sensors. It will also focus to use the more reliable non duplicated communication to ensure the minimum power consumption using sensors. 


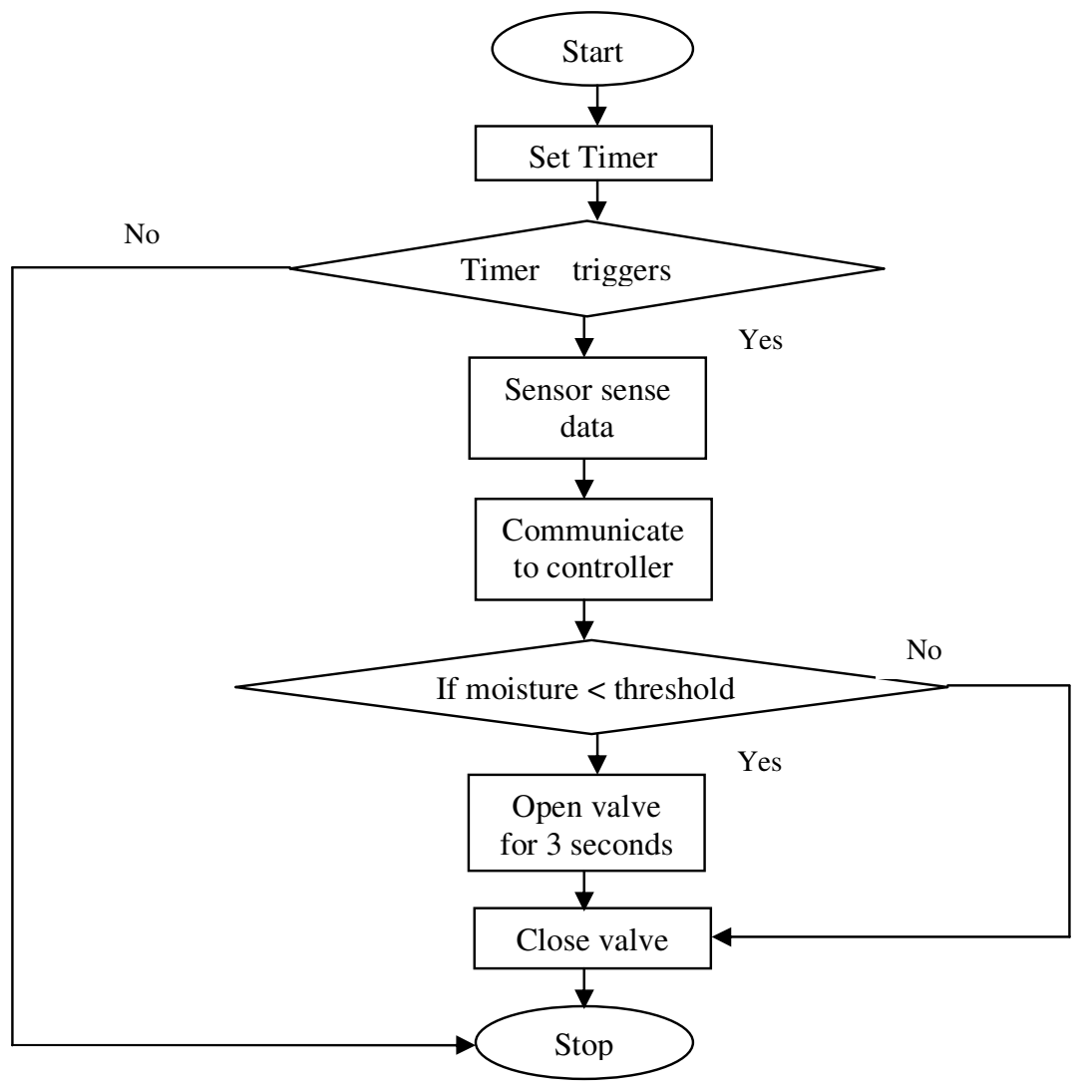

\section{FUTURE RESEARCH}

Agriculture is the process of producing strategic food and fibre in meeting of the fundamental needs. This strategic value has many times been checked over throughout history with the disputes between the nations and environmental limitations. Nowadays where impacts of the global warming come about as the changes/unbalances in the climate conditions sustainability and the increase of the agricultural production is only possible with the collection of more data and analysing and using of them. Detection and the transmission of the data needed are possible with the efficient use of the information technology. It is aimed to distribute the inputs such as fertilisers and chemicals that the plants being grown on the farms need, despite the fact different productivity is obtained in the different regions of the farm or the farms might have different soil structures in the process of conventional agriculture, being integrally taken into account whatever the size of the farm is.

However, the concept of "Sustainable Agricultural Production" that has been put forward aimed at the protection of the environment and the natural resources for the last 15-20 years lays emphasis on the use of these inputs in as less amount as possible and with as much care as possible. In this context, variability in the agricultural production needs to be measured and the inputs need to be applied considering the results. In order to succeed in this process called precision agriculture reliable and continuous data is needed. These days, the environmental data (Temperature, rainfall, humidity etc.), production data on the soil and the plant (Soil nutrition elements, monitoring of the diseases and pests, irrigation). Moreover, it has also been shown that our proposed irrigation model is adaptable for different conditions of weather, land and water. 
International Journal of Ambient Systems and Applications (IJASA) Vol.3, No.2/3, September 2015

\section{Conclusions}

In this paper, a method for developing a smart environment to monitor the irrigational parameter in the entire field is discussed. The system will also focuses on reducing the energy consumption and the cost of communication. It provides a more user friendly way of doing agricultural irrigation. Hence the overall system will pave a way for promoting agriculture in a more automated way.

\section{REFERENCES}

[1] Deepak Dharrao, Laxman Kolape, Sanjeet Pawar, Aniket Patange, "Automated Irrigarion System using WSN", Asian Journal of Engineering and Technology Innovation 03(06); 2015; 18-21.

[2] Liai Gao, Meng Zhang, Geng Chen, "An Intelligent Irrigation System based on Wireless Sensor Network and Fuzzy Control", Journal of Networks, Vol 8, No. 5, May 2013.

[3] M. Damas, A.M. Prados, F. Gomez, G. Oliyares, "HidroBus system: fieldbus for integrated management of extensive areas of irrigated land", Microprocessors and Microsystems 25 (2001) 177184.

[4] Sanbo Li, "Application of the internet of things technology is precision agriculture irrigation systems", International Conference on Computer Science and Service System, 2012.

[5] Nilesh R. Patel, Rahul B. Lanjewar, Swarup S. Mathrukar, Ashwin A. Bhandekar, "Microcontroller based Drip Irrigation System using Smart Sensor", Annual IEEE India Conference (INDICON), 2013.

[6] A. Kumar, K. Kamal, M. O. Arshad, T. Vadamala, S. Mathavan, "Smart Irrigation using Low-Cost Moisture Sensors and XBee based Communication", IEEE 2014 Global Humanitarian Technology Conference.

[7] Ahmed Hussein Abbas, Maya Medhat Mohammed, Gehad Mostafa Ahmed, "Smart Watering System for Gardens using Wireless Sensor Networks", IEEE, 2014.

[8] Chandan Kumar Sahu, Pramitee Behera, "A Low Cost Smart Irrigation Control System", International Conference on Electronics and Communication System (ICECS 2015).

[9] P. Alagupandi, R. Ramesh, S. Gayathri, "Smart Irrigation System for Outdoor Environment using TinyOS", International Conference on Computation of Power, Energy, Information and Communication (ICCEPEIC), 2014.

[10] Mahir Dursun, Semih Ozden, "A Wireless Application of Drip Irrigation Automation supported by Soil Moisture Sensors", Scientific Research and Essays, Vol 6(7), pp.1573-1582, 4 April 2011.

[11] Lutful Karim, Alagan Anpalagan, Nidal Nasser, Jalal Almhana "Sensor-based M2M Agriculture Monitoring Systems for Developing Countries: State and Challenges", Network Protocols and Algorithms, ISSN 1943-3581 2013, Vol. 5, No. 3.

[12] Kim Y., Evans R.G. and Iversen W.M., "Remote Sensing and Control of an Irrigation System Using a Distributed Wireless Sensor Network," Instrumentation and Measurement, IEEE Transactions on, vol.57, no.7, pp.1379-1387, July 2008. http://dx.doi.org/10.1109/TIM.2008.917198

[13] Shock C. C., David R. J., Shock C. A., and Kimberling C. A., "Innovative, automatic, low-cost reading of watermark soil moisture sensors", in Proc. Irrig. Assoc. Tech. Conf., Falls Church, VA, 1999, pp. 147-152.

[14] Miranda F.R., Yoder R., and Wilkerson J.B., "A site-specific irrigation control system", presented at the ASAE Annu. Int. Meeting, Las Vegas, NV, Jul. 27-30, 2003. doi: http://dx.doi.org/10.13031/2013.13740.

[15] Wall R.W. and King B.A., "Incorporating plug and play technology into measurement and control systems for irrigation management", presented at the ASAE/CSAE Annu. Int. Meeting, Ottawa, ON, Canada, Aug. 2004. 
International Journal of Ambient Systems and Applications (IJASA) Vol.3, No.2/3, September 2015

\section{AUTHORS}

Angel C, is an Research Associate from the School of Computing Science and Engineering, VIT University, Chennai. She completed her post graduation in Karunya University, Coimbatore and currently doing her Ph.D in VIT University, Chennai. Her area of interest includes Mobile Computing, Wireless Sensor Networks and Ambient Intelligence.

Dr. S. Asha, is an Associate Professor from the School of Computing Science and Engineering, VIT University, Chennai. She graduated from Madras University, Chennai and completed her Ph.D from Anna University Chennai. Her area of interest includes Network security, Biometric security, Cloud security and Cyber security. She has published many papers in international journal and conferences. Currently she is working in ambient intelligence.

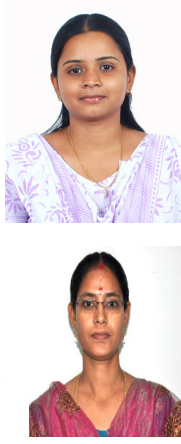

